Revue d'histoire de l'Amérique française

REVUE D.HISTOIRE DE L'AMÉRIQUE FRANÇAISE

\title{
Obligation scolaire et travail des enfants au Québec : 1900-1950
}

\section{Thérèse Hamel}

Volume 38, numéro 1, été 1984

URI : https://id.erudit.org/iderudit/304236ar

DOI : https://doi.org/10.7202/304236ar

Aller au sommaire du numéro

Éditeur(s)

Institut d'histoire de l'Amérique française

ISSN

0035-2357 (imprimé)

1492-1383 (numérique)

Découvrir la revue

Citer cet article

Hamel, T. (1984). Obligation scolaire et travail des enfants au Québec :

1900-1950. Revue d'histoire de l'Amérique française, 38(1), 39-58.

https://doi.org/10.7202/304236ar d'utilisation que vous pouvez consulter en ligne.

https://apropos.erudit.org/fr/usagers/politique-dutilisation/ 


\title{
OBLIGATION SCOLAIRE ET TRAVAIL DES ENFANTS AU QUÉBEC: 1900-1950*
}

\author{
THÉRÈSE HAMEL \\ Institut québécois \\ de recherche sur la culture \\ Québec
}

La conception de la liaison nécessaire de l'enseignement et du travail productif naquit lorsque la grande industrie commença à exploiter le travail des enfants à grande échelle (Kroupskaia (1912), Instruction publique et démocratie, Traduction libre, Université de Paris V, p. 1).

Dans quelque formation sociale que ce soit, ce n'est que sous le capitalisme que naît l'obligation scolaire et seulement après un certain développement de ce mode de production, lié en général à l'apparition de la grande industrie. Pour la première fois dans l'histoire de l'appareil scolaire, tous, enfants d'ouvriers, de paysans, de petits bourgeois et de capitalistes doivent fréquenter l'école pour une période plus ou moins longue, mais où tous et toutes doivent acquérir des connaissances minimales (lire, écrire et compter).

Or, avec le développement du capitalisme et l'une de ses manifestations les plus importantes, la grande industrie, on assiste selon nous à un changement majeur ayant des implications extrêmement importantes sur l'appareil scolaire, le besoin plus généralisé d'une force de travail ayant un minimum de scolarisation.

Ainsi, selon le type d'industrie ou d'entreprise, l'apprentissage devient de moins en moins approprié à la grande industrie, et l'exploitation massive du travail des enfants (qui a été longtemps utilisé grâce à l'allègement du travail dû au développement du machinisme) ne convient plus du tout à certaines fractions de capitalistes, les secteurs d'industrie qu'ils possèdent exigeant une force de travail ayant au moins acquis des connaissances élémentaires: lire-écrire-compter. En obligeant tous les enfants à fréquenter l'école pour une période déterminée comme minimum, l'instruction obligatoire forme un réservoir homogène de travailleurs et travailleuses chez qui il sera possible de puiser les éléments qui pourront ensuite devenir les futurs ouvriers et ouvrières qualifiés.

* Ce texte est tiré de ma thèse de doctorat, L'obligation scolaire au Québec: lieu et enjeu de la lutte des classes (Université de Paris V, René Descartes, 1981). Cette thèse fera peut-être l'objet d'une publication. 
Mais ceci ne se fait pas sans contradictions. D'un côté, le développement des forces productives incite le capitaliste à vouloir une force de travail ayant un minimum de formation. D'un autre côté, l'emploi d'enfants dans la production lui permet de payer des salaires minimes, d'augmenter la population active, de faire ainsi pression sur les salaires, et de réduire au maximum les frais d'entretien de la force de travail, la période préproductive étant de ce fait limitée au maximum. Même si l'exploitation effrénée de la force de travail enfantine met en péril la reproduction même de la force de travail, aucun des capitalistes ne veut cesser l'exploitation de cette main-d'oeuvre bon marché alors que son concurrent la poursuit. Ainsi, les fractions possédant des secteurs à faible besoin de force de travail scolarisée, et à forte consommation de force de travail enfantine come le textile, la chaussure, l'industrie forestière, etc., par exemple, pourront s'opposer à une obligation scolaire qui augmenterait les frais de reproduction d'une main-d'oeuvre ayant des qualifications dont ils n'ont que faire. De plus, elle empêcherait le travail des enfants qui représente une source non négligeable de profit. À l'opposé, d'autres fractions impliquées dans des secteurs de pointe favorisent l'obligation scolaire qui permet la formation d'une force de travail dont ils ont un pressant besoin. Dès la fin du XIX $\mathrm{X}^{\mathrm{e}}$ siècle, des appels dans ce sens ont été formulés par des représentants de cette fraction.

The testimony of not a few proprietors and managers at present employing this class of labor ((children)) lead us to believe that they are willing to discontinue its use, for the benefit of the children, provided that education be compulsory; as demand is gaining for intelligent and educated labor in our mills and factory. ${ }^{p}$

L'obligation scolaire permet de résoudre les contradictions de la bourgeoisie en éliminant pour tous les capitalistes le marché de la force de travail enfantine, les mettant tous sur le même pied dans la concurrence inter-capitaliste. De plus elle permet de former la main-d'oeuvre à un coût moindre que si chaque capitaliste formait lui-même sa force de travail. Elle est aussi le résultat des luttes de la classe ouvrière pour l'abolition du travail des enfants et l'instauration de la fréquentation scolaire obligatoire $^{2}$.

1 «Le témoignage de plusieurs propriétaires et directeurs qui emploient actuellement ce type de travailleurs (les enfants, T.H.) nous porte à croire qu'ils veulent complètement en arrêter l'usage au profit des enfants, pourvu que l'instruction soit obligatoire; étant donné que la demande de main-d'oeuvre intelligente et instruite augmente dans nos usines et fabriques (traduction de l'auteur).» Report of the Commissionners appointed to enquire into the Working of Mills and Factories of the Dominion (1882), dans D. Lawr, R. Gidney (éd.), Educating Canadians (Toronto, Van Nostrand Reinhold Ltd., 1973), 66.

2 Pour une articulation plus complète des enjeux de l'instruction obligatoire pour l'Église et les classes ouvrières et paysannes, nous renvoyons les lecteurs et lectrices à Thérèse Hamel, L'obligation scolaire au Québec: lieu et enjeu de la lutte des classes (Université de Paris V, René Descartes, 1981), 448 pages. 
Quoique née sous le capitalisme, l'obligation scolaire arrive à des moments très différents selon les formations sociales. Acquise dès 1882 en France et en 1891 en Ontario, il faut attendre 1943 au Québec, pour que soit votée la loi de fréquentation scolaire obligatoire (7 Geo. VI), alors qu'aux États-Unis, dès 1918, tous les États ont une loi d'obligation scolaire, dont certaines datent même de la fin du XIX ${ }^{\mathrm{e}}$ siècle. Au Québec, la lutte pour l'obligation scolaire dure plus de cinquante ans. Dès 1892, un premier projet de loi est présenté à l'Assemblée nationale. Celui-ci est refusé. Suivent les projets de 1901, de 1912 (par et pour les protestants), le long débat de 1919 et enfin le vote de la loi en 1943. L'avènement de l'obligation scolaire au Québec s'inscrit dans un tissu de relations très complexes, d'alliances et de luttes entre la classe ouvrière (divisée en fractions), l'Église et certaines fractions de la bourgeoisie. D'une part, une fraction de la bourgeoisie se trouve du même côté que la fraction de la classe ouvrière représentée par le Congrès des métiers et du travail du Canada (CMTC) qui approuve l'instruction obligatoire. En face, l'Église farouchement opposée à l'obligation scolaire, s'assure de l'appui d'une partie de la classe ouvrière et de la classe agricole, regroupées respectivement au sein de la Confédération des travailleurs catholiques du Canada (CTCC) et de l'Union catholique des cultivateurs (UCC). Pourquoi un tel retard au Québec, tant par rapport à l'Europe qu'au reste de l'Amérique du Nord?

Un des facteurs permettant d'expliquer le retard du vote de la loi de fréquentation scolaire obligatoire est l'utilisation plus tardive au Québec du travail des enfants, par rapport au reste du Canada. En effet, l'exploitation de la très jeune force de travail au Canada s'est concentrée de plus en plus au Québec, et ceci jusque dans les années quarante. La présence au Québec d'un important secteur industriel à faible besoin de force de travail scolarisée et à forte consommation de jeunes bras, et la prédominance de la ferme familiale d'auto-subsistance à forte utilisation de la main-d'oeuvre familiale, expliquent en partie ce phénomène ${ }^{3}$.

Un autre facteur permettant d'expliquer le retard de l'avènement de l'obligation scolaire est la force qu'a conservée l'Église au Québec, jusque dans les années cinquante, force qui s'est opposée farouchement à l'obligation scolaire.

Enfin, le mouvement ouvrier, syndical et populaire, quoique ayant lutté pour l'obligation scolaire, a eu d'énormes difficultés à faire pencher le rapport de force dans le sens de l'obligation scolaire, à cause de grandes divisions en son sein.

\footnotetext{
3 Étant données les limites de ce court texte, nous n'aborderons ici que le rapport entre le retard de l'obligation scolaire et le travail des enfants, pour montrer comment ce facteur a eu sa part de responsabilité dans le retard de l'obligation scolaire au Québec.
} 


\subsection{Fréquentation scolaire et travail des enfants}

\subsection{Législation sur le travail des enfants et la fréquentation scolaire obligatoire}

L'étude de la législation sur le travail des enfants et la fréquentation scolaire obligatoire nous donne déjà un indice des rapports sociaux à cette époque. En effet, même si l'analyse d'une loi ne permet pas de cerner ce qu'en fut l'application réelle, elle nous indique le sens des luttes qui ont présidé à son avènement. Avant d'aborder directement la position de la législation québécoise par rapport à celle du reste du Canada, il convient de montrer le lien étroit qui existe entre la législation sur le travail des enfants et celle sur la fréquentation scolaire obligatoire. À cause de dures luttes de la classe ouvrière et parce que la bourgeoisie a fini par reconnaître la mise en péril de la reproduction de la force de travail due à l'exploitation massive des enfants, on a commencé par légiférer sur l'augmentation de l'âge minimum d'entrée au travail, et sur la diminution des heures de travail.

Alors qu'à la fin du siècle, on tente de réduire le travail des enfants pour des raisons de santé et à cause des demandes incessantes de la classe ouvrière, on en vient ensuite à parler d'instruction pour les enfants qui travaillent, de façon à améliorer leur productivité. Ainsi, un manque de formation minimum de la force de travail devient-il un handicap pour une fraction du Capital. Alors qu'auparavant le travail des enfants était directement rentable, le développement des forces productives oblige à une formation minimum de la force de travail dans certains secteurs. Avant qu'une loi de fréquentation scolaire obligatoire puisse mettre fin à la concurrence inter-capitaliste face au travail des enfants, on passe par une période intermédiaire où on exige un certain niveau de connaissances, tout en continuant à exploiter la jeune force de travail. Un exemple de ceci est l'organisation des cours du soir pour les enfants, qui tout en donnant la formation nécessaire, le soir, permettent l'exploitation massive des enfants le jour.

À cet égard, il est significatif de voir qu'aux États-Unis, les capitalistes demandent, à la fin du siècle dernier, que si l'école devient obligatoire, ce soit le soir ${ }^{4}$. L'école du soir est pour nous l'indice d'un besoin de force de travail ayant un minimum de connaissances, dû au développement des forces productives, dans une conjoncture où le rapport de force n'est pas encore favorable à l'imposition par la classe ouvrière, de l'obligation scolaire. On passe donc par une période où l'exploitation des enfants n'est freinée par aucune loi, suivie d'une période intermédiaire où on allie exploitation des enfants le jour et

4 F.C. Ensign, Compulsory school attendance and child labor (Iowa City, Athen Press, 1969, réédition de 1921). 
formation de la force de travail, le soir, pour finalement en arriver à l'obligation scolaire.

Ainsi, les deux types de législation s'imbriquent, l'obligation scolaire s'avérant un moyen très efficace d'enrayer le travail des enfants. En effet, alors que les lois sur le travail des enfants ne touchent que ceux et celles considérés comme étant sur le marché du travail ${ }^{5}$, les lois d'obligation scolaire obligent tous les enfants à fréquenter l'école pour une période donnée.

Alors que dans une formation sociale à juridiction unique, une loi sur le travail des enfants permet de mettre tous les capitalistes sur le même pied, au Canada, à cause de la double juridiction fédérale et provinciale et dû au fait que le travail des enfants et la fréquentation scolaire obligatoire soient de juridiction provinciale, il peut y avoir de fortes différences d'une province à l'autre quant aux possibilités d'utiliser les enfants dans la production. Un parcours rapide de la législation canadienne permettra de voir que le Québec s'avérait un foyer propice à l'exploitation du travail des enfants.

\subsection{Tentatives de limitation de la concurrence inter-capitalistes au niveau législatif}

À plusieurs reprises, des projets de loi ont été présentés dans le but de réglementer tant le travail des enfants que la fréquentation scolaire obligatoire au niveau pan-canadien, de façon à limiter la concurrence inter-capitaliste. En 1882, un projet de loi est présenté au Sénat par James C. Aikins, dans le but d'exiger dix ans comme âge minimum d'emploi dans les fabriques et de limiter le travail des moins de quatorze ans à huit heures par jour ou trente heures par semaine. En 1884, en Ontario, sir Leonard Tilley propose que les infractions aux lois sur le travail des enfants soient de juridiction criminelle (c'est-à-dire dépendant du fédéral), ce qui est déclaré anticonstitutionnel à cause des juridictions provinciales. En 1889, la Commission royale d'enquête sur les relations entre le capital et le travail insiste sur les avantages que procurent à certains capitalistes les différences entre les législations provinciales et fédérales. Ces domaines n'en sont pas moins restés de juridiction provinciale, mais ces luttes indiquent que lorsque une fraction de la bourgeoisie profite du travail des enfants, une autre s'estime lésée par la concurrence exercée grâce au travail des enfants sous-payés.

Des projets semblables ont été présentés dans le but d'uniformiser les lois portant sur la fréquentation scolaire.

5 Nous verrons plus loin les secteurs non touchés par la loi. 


\subsection{Où déjà dans la législation le Québec s'avère un foyer propice à l' exploitation de jeunes enfants ${ }^{6}$}

Nous examinerons la législation principalement sous deux aspects: l'âge minimum d'entrée au travail et l'exigence de certaines habiletés scolaires (entre autres lire, écrire, compter), qu'elles soient incluses dans les lois sur le travail des enfants ou sur la fréquentation scolaire obligatoire.

Le Tableau 1 nous donne les principales lois limitant le travail des enfants au Québec.

Lorsque l'on compare la législation québécoise sur le travail des enfants à celle des autres provinces, on constate que l'âge minimum d'emploi s'élève moins rapidement au Québec. Il faut attendre 1907 au Québec pour que quatorze ans soit l'âge minimum d'emploi, alors que c'est déjà le cas en Ontario depuis 1895 (sauf dans les conserveries), depuis 1900 au Manitoba, depuis 1901 en Nouvelle-Écosse (sauf dans les conserveries de juillet à octobre) et depuis 1905 au Nouveau-Brunswick (ne s'appliquant pas hors des villes). Dans les provinces où quatorze ans n'est pas encore l'âge minimum d'emploi, la loi sur la fréquentation scolaire obligatoire apporte certaines limitations au travail des enfants. Le Québec ne possède à cet égard que les lois sur le travail des enfants pour en limiter l'exploitation.

Plus significatif encore, le Québec, qui ne possède aucune loi sur l'instruction obligatoire, est aussi une des provinces où les exigences de formation minimale de la force de travail (lire, écrire, compter) incluses dans les lois sur le travail des enfants sont les plus faibles.

Ainsi, pour ce qui est des mines, contrairement aux autres provinces qui dès la fin du $\mathrm{XIX}^{\mathrm{e}}$ siècle exigent un certain niveau scolaire pour travailler, ou ont une loi de fréquentation scolaire obligatoire, le Québec n'a aucune exigence à ce sujet. En Nouvelle-Écosse, par exemple, en 1891, les enfants ayant entre douze et dix-huit ans doivent savoir lire, écrire et faire des opérations arithmétiques simples.

Dans les fabriques et usines, au Québec, la première exigence de formation est incluse dans la loi de 1907 où il est requis des enfants de moins de seize ans qu'ils sachent lire et écrire ou qu'ils fréquentent les cours du soir, alors que dès le dernier quart du XIX ${ }^{e}$ siècle, plusieurs provinces ont déjà décrété l'obligation scolaire pour les enfants d'un certain âge (en général de 7 à 12 ans) et pour une période minimale de l'année. La première province à voter l'obligation scolaire pour l'année entière est l'Ontario où la fréquentation scolaire obligatoire de huit à quatorze ans est exigée dès 1891 . À la fin de la première guerre mon-

6 Cette partie est inspirée, pour les données, de: Gouvernement du Canada, Department of Labour, The employment of children and young persons in Canada (Ottawa, 1930), 90-133. 
TABLEAU 1

LOIS LIMITANT LE TRAVAIL DES ENFANTS AU QUÉBEC

1885 (C.32): Pour les entreprises de 20 employés et plus.

Âge minimum: 12 ans - garçons

$$
14 \text { ans - filles }
$$

Certificat d'âge exigé pour les moins de 14 ans

1888 (C.49): La loi précédente s'applique à toutes les fabriques et usines.

1892 (C.20): Pour les mines seulement.

Âge minimum: 15 ans pour les garçons pour le travail sous terre.

Défendu aux filles.

Aucun âge minimum pour les mines métallifères.

1903 (C.30): Dans les fabriques et usines.

Âge minimum élevé à 13 ans pour les garçons et 14 ans pour les filles.

1907 (C.39): Âge minimum: 14 ans pour les garçons et filles.

Exigence de formation: savoir lire et écrire pour les moins de 16 ans ou fréquentation des cours du soir.

1919 (C.50): Âge minimum: 16 ans pour ceux qui ne savent pas lire ni écrire couramment, mais l'inspecteur peut donner un permis de travail si la personne fréquente les cours du soir.

1934 (C.35): Les magasins sont incorporés dans la législation sur les établissements industriels.

SOURCE: Gouvernement de la province de Québec, Statuts du Québec.

diale, le Québec est la seule province à ne pas avoir de loi d'instruction obligatoire, à une époque où tous les États américains l'exigent.

Ce bref aperçu de la place de la législation québécoise par rapport au reste du Canada permet de voir très clairement que le Québec, tout en étant la province où la fréquentation scolaire est votée le plus tard (1943), est aussi celle où les lois sur le travail des enfants présentent le moins d'exigences sur le plan des habiletés minimales, d'autant plus qu'elles ne sont pas renforcées par une législation sur la fréquentation scolaire obligatoire.

Nous venons de voir comment, dans la législation canadienne, le Québec s'avère un foyer propice à l'exploitation de jeune force de travail. Qui plus est, les mesures visant à l'application de la loi sont pour le moins modestes. La première loi des établissements industriels est votée en 1885. Il faut attendre trois ans pour que les premiers inspecteurs soient nommés. De plus, les inspecteurs sont trop peu nombreux devant l'ampleur du travail à accomplir. En 1888, ils ne sont que trois pour couvrir l'ensemble du territoire de la province de Québec. On parle de certificats d'âge et de certificats d'études à quelques reprises, mais les rapports des inspecteurs de fabriques regorgent d'exemples de 
ruses pour contourner la loi: faux certificats d'âge, enfants qui se cachent à la venue de l'inspecteur, utilisation du même certificat pour plus d'un enfant, etc... Enfin, les lois permettent de longues heures de travail aux enfants (10 heures par jour, pouvant aller jusqu'à 12 heures par jour pendant au plus six semaines selon la loi de 1885; 58 heures/semaine dans les filatures de coton pour les enfants de moins de dix-huit ans, selon la loi de 1907), ce qui en dit long sur les conditions d'exploitation de la jeune force de travail.

Mais de plus, et ceci sans compter la non application des lois, de graves omissions ou restrictions dans la législation existante permettent de disposer de jeunes bras dociles et sous-payés. Ainsi, aucune législation au niveau canadien ne concerne l'agriculture, qui constitue pourtant un secteur très important de l'activité productive et qui, dans le cas précis du Québec, concentre longtemps la majorité de la force de travail. Pour ce qui est du secteur commercial, il faut attendre 1934 au Québec (contrairement à l'Ontario en 1913) pour que les commerces entrent dans le champ de la loi sur les établissements industriels. Toutefois, la loi de 1919 obligeant les personnes de moins de seize ans à savoir lire et écrire ou à fréquenter les cours du soir, s'applique aussi aux établissements commerciaux. En 1903, Louis Guyon, chef des inspecteurs de fabrique au Québec disait:

It must not be forgotten that the employees in the majority of our shops are chiefly children (...) shops should have been brought under the Industrial Establishment Act so as to allow our intervention. ${ }^{7}$

Ce secteur d'emploi, de même que le travail dans la rue, fut le moins contrôlé, alors qu'il est très facile d'y employer des enfants, tant en dehors des heures de classe que durant l'horaire scolaire. Enfin, le travail domestique, qui occupe une bonne partie de la force de travail féminine, ne fait l'objet d'aucune législation, et celle sur le travail des enfants ne concerne pas les entreprises familiales non mécanisées. Le Québec s'avère donc déjà, au niveau législatif, propice à l'exploitation du travail des enfants, et de plus, les lois existantes sont d'une extrême «souplesse», ceci sans parler des conditions de travail, des longues journées, des accidents du travail, etc., etc.

\footnotetext{
«Il ne faut pas oublier que les employés dans la plupart de nos magasins sont en majorité des enfants (...) les magasins devraient être sous la juridiction de la loi des établissements industriels pour que nous puissions intervenir (traduction de l'auteur).» Gouvernement de la province de Québec, Annual Report of the Quebec Department of Labour (1903); dans Terry Copp, The anatomy of poverty. The Condition of the Working Class in Montreal, 1897-1929 (Toronto, McClelland and Stewart, 1974), 52.
} 


\subsection{Fréquentation scolaire au Québec avant 1943}

Nous allons maintenant étudier la fréquentation scolaire des enfants à l'aide des recensements fédéraux de 1911 à 1941 inclusivement. Comme la fréquentation scolaire obligatoire entre en compétition avec le travail des enfants, c'est donc surtout la fréquentation scolaire entre dix et quatorze ans qui nous intéressera plus particulièrement.

L'examen de la fréquentation scolaire de la population de sept à quatorze ans, pour le Canada et les provinces, de 1911 à 1941, nous montre que le Québec est la province ayant le taux de fréquentation scolaire le plus bas en 1931 et 1941. En 1921, elle suit le NouveauBrunswick, qui est, avec le Québec, la province où la législation sur la fréquentation scolaire obligatoire est la plus souple. Quoique les données pour le Québec en 1911 montrent un taux relativement satisfaisant de fréquentation scolaire $(80,92 \%)$, ces chiffres ne donnent qu'une vision très partielle de la réalité. En effet, selon la question du recensement ${ }^{8}$, une personne ayant fréquenté l'école à la limite une journée était considérée comme ayant fréquenté l'école cette année-là. Il n'en reste pas moins que, malgré une amélioration de décennie en décennie, 10,62\% des enfants de sept à quatorze ans ne fréquentaient pas l'école du tout en 1931 , et encore $8,4 \%$ en 1941 , soit deux ans avant la promulgation de la loi.

Examinons de plus près la situation de ceux et celles qui sont recensés comme fréquentant l'école. Demeurent-ils longtemps dans l'appareil scolaire? Lorsque l'on compare les cas du Canada, de l'Ontario et du Québec, pour les années 1911, 1921, 1931 et 1941, pour ce qui est de la fréquentation scolaire âge par âge, il est frappant de constater que malgré un niveau de fréquentation scolaire presque aussi élevé au Québec qu'au Canada et qu'en Ontario pour le groupe d'âge de 7 à 12 ans, on observe une chute très brusque de cette fréquentation entre 12 et 13 ans et entre 13 et 14 ans au Québec. En Ontario, il existe aussi une coupure, mais qui s'effectue entre 13 et 14 ans, et encore là, les taux de fréquentation y sont beaucoup plus élevés qu'au Québec. Alors qu'à l'âge de douze ans en 1911, les taux sont respectivement de 84,0\%, $84,6 \%$ et $88,3 \%$ au Canada, au Québec et en Ontario, à treize ans, le Québec ne conserve que 73,6\% des enfants de cet âge à l'école, alors qu'en Ontario, 83,6\% sont encore dans le système scolaire. À quatorze ans au Québec, il ne reste qu'un peu plus de la moitié des enfants de cet âge à l'école, $54,5 \%$ contre 68,4\% en Ontario et 63,3\% au Canada.

Plus on avance dans le temps et plus l'écart entre le Québec et l'Ontario s'accroît. En 1931, à quatorze ans, le Canada conserve 83,3\% de la population de cet âge dans l'appareil scolaire et l'Ontario 90,4\%.

\footnotetext{
$8 \quad$ Il était demandé si la personne avait fréquenté une institution d'enseignement pendant une période quelconque durant l'année scolaire de recensement.
} 
Au même moment, au Québec, le pourcentage tombe à 67,7\%, c'està-dire un écart de 22,7 points par rapport à l'Ontario.

Le Québec se caractérise donc par une fréquentation scolaire beaucoup plus courte qu'en Ontario et qu'au Canada, fréquentation qui fléchit massivement entre 12 et 13 ans et entre 13 et 14 ans.

Quand on analyse les variations de la fréquentation scolaire âge par âge, selon le milieu rural et urbain, au Québec et en Ontario, on constate d'abord une tendance commune: on quitte plus tôt l'école en milieu rural qu'en milieu urbain, mais le Québec se caractérise par une désertion beaucoup plus hâtive qu'en Ontario, et par des taux de fréquentation beaucoup plus bas. Alors qu'à 13 ans en 1921, la fréquentation scolaire en milieu rural est encore de 90,3\% en Ontario, au Québec elle n'est plus que de $74,1 \%$, différence énorme. À 14 ans, il ne reste que 49,3\% des enfants de cet âge à l'école en milieu rural au Québec, contre 73,8\% en Ontario. En 1931, en milieu rural, le Québec ne conserve que $52,1 \%$ des enfants de 14 ans à l'école, contre $82,3 \%$ en Ontario. Enfin, en 1941, alors qu'en Ontario en milieu rural, on retrouve 79,7\% des enfants de 14 ans à l'école, au Québec, il n'en reste que $56,2 \%$.

La chute très brusque de la fréquentation scolaire à partir de douze ans est très significative et c'est ce facteur qui caractérise principalement le Québec: l'abandon précoce de l'appareil scolaire. Même sans loi de fréquentation scolaire obligatoire, il y avait donc fréquentation scolaire mais l'école ne retenait les enfants que quelques années. Un des enjeux de l'instruction obligatoire est donc de prolonger la fréquentation scolaire.

\subsection{Concentration de la non-fréquentation scolaire canadienne au Québec}

L'étude de la non-fréquentation scolaire au Canada et dans les provinces, en chiffres absolus, nous permet de montrer de facon très claire le rôle que le Québec joue dans la concurrence inter-capitaliste pour ce qui est du marché du travail des enfants. En effet, entre 1911 et 1941, le Québec concentre de plus en plus le nombre de jeunes de sept à quatorze ans qui ne vont pas à l'école au Canada.

En 1911, le Québec concentre 30,4\% des jeunes ne fréquentant pas une institution scolaire canadienne. La proportion s'élève à $35,2 \%$ en 1921 , à $46,1 \%$ en 1931 pour atteindre $47,1 \%$ en 1941 . Pendant ce temps, en Ontario, les pourcentages diminuent progressivement passant de $25,5 \%$ en 1911 à $18,7 \%$ en 1931 , pour augmenter très légèrement en 1941, passant à 20,1\%. Ainsi, l'écart entre le Québec et l'Ontario augmente-t-il constamment. 
La chute de la fréquentation scolaire s'effectue après l'âge de dix ans. C'est aussi à partir de cet âge que les enfants sont susceptibles d'être «happés» par le travail salarié. Or, la concentration de la nonfréquentation scolaire au Québec est encore plus flagrante pour ce groupe d'âge.

Alors que l'on ne retrouve au Québec en 1921 que 30,1\% de la population canadienne de 10 à 14 ans, 40,9\% de la non-fréquentation scolaire y est concentrée. Ce pourcentage ira croissant par la suite pour se stabiliser autour de 50\% jusqu'en 1951.

Plus on avance dans le temps, et quel que soit l'âge choisi, on observe une augmentation de la concentration de la non-fréquentation scolaire canadienne au Québec, concentration qui atteint 60,6\% en 1931, à l'âge de 13 ans. C'est-à-dire que parmi tous les enfants de treize ans qui ne fréquentent pas l'école au Canada en 1931, 60,6\% sont au Québec. En Ontario, on assiste à une diminution de la proportion canadienne de la non-fréquentation scolaire. Enfin, c'est à 13 et 14 ans que le Québec concentre massivement la non-fréquentation scolaire canadienne, les pourcentages allant jusqu'à $56,7 \%$ en 1931, pour l'âge de 14 ans. Seule province sans loi de fréquentation scolaire obligatoire, le Québec concentre donc de plus en plus la non-fréquentation scolaire canadienne. Ce phénomène est d'autant plus marqué que l'âge des enfants tend vers 14 ans.

\subsection{Travail des enfants}

Quelle part de cette non-fréquentation scolaire peut être expliquée par le travail des enfants?

À l'aide des recensements fédéraux, nous ne pouvons retracer qu'une partie des enfants qui travaillent. Quoique déjà très révélateurs de l'acuité du problème, les recensements, de la façon dont ils sont construits, ne donnent qu'une image tronquée de la réalité. Ainsi, dans les recensements:

Les enfants de dix ans et plus employés aux travaux du ménage chez leurs parents ou aux travaux de la ferme, ou à tout autre travail ou tâche, quand ils ne fréquentent pas l'école, ne doivent pas être inscrits comme ayant un emploi. ${ }^{9}$

De plus, on n'a pas recensé comme travail, le travail domestique des filles, le travail à la ferme des enfants, sauf lorsque ce travail constitue une occupation majeure dans l'année, le travail des enfants de plus de dix ans en dehors des heures de classe ou durant une partie de l'année comme le travail forestier, l'aide aux récoltes.

\footnotetext{
9 Gouvernement du Canada, Recensement du Canada (Ottawa, 1921), IV, p.c. (le souligné est de nous).
} 
Nous verrons plus loin les conséquences de telles omissions, ceci sans compter la non-application de la loi, les enfants employés illégalement, les enfants non recensés etc... C'est donc dire que ces chiffres, quoique très révélateurs, ne montrent qu' une partie de l'exploitation du travail des enfants.

\subsection{Concentration du travail des enfants au Québec}

Nous avons vu comment le Québec concentrait la non-fréquentation scolaire. Nous ne serons pas surpris qu'il concentre aussi le travail des enfants au Canada. Le Tableau 2 nous montre le nombre d'enfants de 10 à 14 ans qui travaillent en $1891,1911,1921$ et $1931^{10}$.

En 1891, l'Ontario concentre $44,5 \%$ du travail des enfants au Canada et le Québec $29,1 \%$. En 1911, les pourcentages des deux provinces se rapprochent, $32,9 \%$ pour le Québec et $40,2 \%$ pour l'Ontario. À partir de 1921, le Québec concentre presque 50\% du travail des moins de quatorze ans au Canada, contre seulement $24,2 \%$ en Ontario. De plus, il concentre 54\% du travail des enfants de 10 à 13 ans. En 1931, les proportions atteignent $60,4 \%$ au Québec contre à peine $14,2 \%$ en Ontario. Enfin, le Québec concentre 56,9\% en 1941 et 48,9\% en 1951 du travail des enfants de quatorze ans contre $24,1 \%$ et $24,7 \%$ en Ontario $^{11}$. Ainsi, plus on avance dans le temps et plus le Québec s'avère un foyer de concentration du travail des enfants canadiens.

TABLEAU 2

TRAVAIL DES ENFANTS DE 10 À 14 ANS, EN CHIFFRES ABSOLUS ONTARIO, QUÉBEC ET CANADA 1891, 1911, 1921, 1931

\begin{tabular}{|c|c|c|c|c|c|c|}
\hline \multirow[b]{2}{*}{1891} & \multicolumn{2}{|c|}{ ONTARIO } & \multicolumn{2}{|c|}{ QUÉBEC } & \multicolumn{2}{|c|}{ CANADA } \\
\hline & 33635 & $44,5 \%$ & 21962 & $29,1 \%$ & 75601 & $100 \%$ \\
\hline 1911 & 10102 & $40,2 \%$ & 8275 & $32,9 \%$ & 25153 & $100 \%$ \\
\hline 1921 & 7150 & $24,2 \%$ & 14104 & $47,7 \%$ & 29566 & $100 \%$ \\
\hline 1931 & 3111 & $14,2 \%$ & 11536 & $60,4 \%$ & 19113 & $100 \%$ \\
\hline
\end{tabular}

SOURCES: 1891, 1911, 1921: Gouvernement du Canada, Department of Labour, The employment of children and young persons in Canada (Ottawa, 1930): 17. - 1931: Gouvernement du Canada, Recensement du Canada (Ottawa, 1931), VII: 696-709.

\footnotetext{
10 Malheureusement, les données fournies par les recensements fédéraux ne nous permettent pas de donner un tableau complet du travail des enfants de 1891 à 1951, les groupes d'âge utilisés variant d'une décennie à l'autre. Nous devrons travailler avec les catégories suivantes: 10 à 14 ans pour $1891,1911,1921$ et $1931 ; 10$ à 15 ans pour 1901,1921 et $1931 ; 14$ et 15 ans pour 1941 et 1951 .

${ }_{11}$ Les chiffres pour ces deux années ne sont donnés que pour cet âge seulement.
} 
Les enfants de 10 à 14 ans qui travaillent représentent en 1911 $4,3 \%$ de la population de cet âge en Ontario contre 3,7\% au Québec. En 1921, au Québec, ils représentent $5,1 \%$ de la classe d'âge contre $2,6 \%$ en Ontario et en 1931 , alors qu'ils représentent $3,7 \%$ de la classe d'âge au Québec, en Ontario ils ne forment que 1\% du groupe ayant de 10 à 14 ans.

Quoique très significatifs de l'ampleur du problème du travail des enfants au Québec, ces chiffres sont biaisés à la baisse, du fait qu'ils incluent les enfants de 10 et 11 ans qui eux ne constituent qu'une infime partie de la force de travail, étant donné qu'à cet âge, l'on fréquente encore relativement bien l'école. L'examen du groupe de quatorze ans est encore plus éclairant sur l'importance du travail des enfants au Québec. Malheureusement, il est impossible d'isoler pour 1931 la population de quatorze ans qui travaille. Néanmoins, nous disposons de données tant pour 1921 que pour 1941.

En 1921, les enfants de quatorze ans qui travaillent représentent 17,5\% de la classe d'âge au Québec contre $11,8 \%$ au Canada et 10,7\% en Ontario. En 1941, deux ans avant le vote de la loi sur la fréquentation scolaire obligatoire, pas moins de $10,0 \%$ des enfants de quatorze ans faisaient partie de la population active (tel que recensé par le gouvernement fédéral). La proportion est de $4,7 \%$ en Ontario et de 5,7\% au Canada.

L'absence de loi de fréquentation scolaire obligatoire et une législation sur le travail des enfants très «complaisante» rendent donc le Québec apte à jouer le rôle de réservoir de jeunes forces de travail au niveau pan-canadien.

\subsection{Garçon ou fille?}

Ceci dit, comment se répartissent les enfants recensés comme travaillants? On remarque d'abord une très grande disparité entre les garçons et les filles (Tableau 3). Le tableau montre la prépondérance des garçons recensés comme travaillant par rapport aux filles du même âge.

Les définitions de recensement expliquent en partie le phénomène. En effet, sont considérées comme travaillant les personnes qui consacrent la majeure partie de leur temps à des tâches autres que le ménage, activités habituellement réservées aux filles!

\footnotetext{
12 Les chiffres de 1911 sont, de l'avis de A. Raynauld, sous-estimés, et principalement ceux du travail des enfants masculins de 10 à 14 ans. Dans Croissance et structure économique de la province de Québec, (Ministère de l'Industrie et du Commerce, 1961), 566.
} 
TABLEAU 3

RÉPARTITION DU TRAVAIL DES ENFANTS DE 10 À 14 ANS SELON LE SEXE, QUÉBEC, 1891, 1911, 1921, 1931 ${ }^{12}$

\begin{tabular}{lrrrrrrrrr}
\hline & \multicolumn{2}{c}{ Masculin } & $\%$ & & \multicolumn{2}{c}{ Féminin } & $\%$ & & \multicolumn{2}{c}{ Total } & $\%$ \\
\cline { 2 - 5 } \cline { 8 - 9 } 1891 & 20287 & 92,4 & & 1675 & 7,6 & & 21962 & 100 \\
1911 & 5603 & 67,7 & & 2672 & 32,3 & & 8275 & 100 \\
1921 & 11853 & 84,0 & & 2251 & 16,0 & & 14104 & 100 \\
1931 & 9964 & 86,3 & & 1572 & 13,6 & & 11556 & 100 \\
\hline
\end{tabular}

SOURCES: 1891, 1911, 1921: Gouvernement du Canada, Department of Labour, The employment of children and young persons in Canada (Ottawa, 1930): 17. - 1931: Gouvernement du Canada, Recensement du Canada (Ottawa, 1931), VII: 696-709.

\subsection{Où travaillent ces enfants?}

De plus, le travail des enfants se concentre dans des secteurs économiques bien précis ${ }^{13}$.

Les trois secteurs qui utilisent le plus le travail des enfants sont l'agriculture, les services et les manufactures. À part 1911, où la distribution est plus dispersée, et où les manufactures surclassent les services domestiques et l'agriculture (respectivement 40,3\%, 17,4\% et $14,2 \%$ du travail des enfants), l'agriculture est de loin le premier employeur de jeune force de travail (69,9\% des enfants en 1921, 68,3\% en 1931 , et $72,8 \%$ en 1941). Les services suivent avec $17,4 \%$ des enfants en $1911,8,9 \%$ en $1921,11,7 \%$ en 1931 et $10,8 \%$ en 1941 . Les manufactures, qui retenaient $40,3 \%$ des enfants en 1911 n'en gardent plus que $9,7 \%$ en $1921,7,4 \%$ en 1931 et $5,7 \%$ en 1941 (après le commerce, $6,8 \%)$.

Lorsque l'on examine l'emploi des enfants selon le sexe, on remarque qu'environ $80 \%$ des garçons travaillent massivement dans l'agriculture et qu'entre 60 et $80 \%$ des filles sont dans les services (domestiques) entre 1921 et 1941, ce qui ne surprend guère.

Il n'y a qu'en 1911 que les manufactures dépassent ces secteurs traditionnels, employant $35,1 \%$ des garçons contre $20,9 \%$ pour l'agri-

13 Cette partie se base sur les données suivantes: Gouvernement du Canada, Recensement $d u$ Canada (Ottawa, 1911), VI: 12-13; VI: 206-208; (1921), IV: 188-215; (1931), VII: 696-703, VII: 46; (1941), VII: 570-585. Malheureusement, les groupes d'âge varient selon les recensements: 1911 et $1921-10$ à 13 ans; $1931-10$ à 14 ans; $1941-14$ ans. Pour 1891 et 1901, nous n'avons que la distribution dans les manufactures, ce qui n'inclut qu'une fraction du travail des enfants. 
culture et $51,1 \%$ des filles contre $42,2 \%$ pour les services. Ce sont les manufactures de coton qui étaient les principales employeuses ${ }^{14}$.

Nous venons de voir qu'en ne considérant qu' une partie seulement du travail des enfants, les recensements fédéraux permettent de cerner l'ampleur du problème dans la province de Québec. On peut déjà en tirer plusieurs conclusions.

Plus on avance dans le temps, plus le Québec concentre une proportion élevée du travail des enfants canadiens, allant jusqu'à $60,4 \%$ en 1931. Avant le vote de la loi de fréquentation scolaire obligatoire en 1943, 7051 personnes de quatorze ans au Québec étaient considérées comme ayant un emploi rémunéré, ce qui représente $10 \%$ de la population de cet âge. Enfin, l'agriculture concentre le travail des garçons alors que les filles travaillent surtout dans les services.

\subsection{Travail des enfants en dehors des recensements}

Mais tous ces chiffres, quoique très éloquents, ne nous montrent que la partie visible de l'iceberg. En effet, tout le travail domestique effectué par les filles à la maison est totalement évacué dans les recensements. Alors que les garçons effectuant un travail non rémunéré pour leurs parents à la ferme sont considérés comme actifs, les filles aidant aux travaux de ménage sans recevoir de rémunération ne sont pas considérées comme faisant partie de la population active. Lorsque l'on additionne la proportion des garçons de quatorze ans à l'école $(60,53 \%)$ et ayant un emploi rémunéré recensé $(28,49 \%)$, on retrouve $89,02 \%$ des garçons en 1921. Si l'on accomplit la même opération chez les filles, on ne retrace que $67,35 \%$ d'entre elles $(60,98 \%$ à l'école et $6,37 \%$ ayant un emploi recensé $)^{15}$. Ni à l'école, ni calculées comme faisant partie de la population active, les filles sont de fait à la maison, effectuant les tâches ménagères qui ne sont pas considérées comme du travail (entendu rémunéré). De plus, cette absence des enfants, tant à l'école que directement dans la production, s'explique par l'utilisation des enfants de dix à treize ans comme gardiens de leurs frères et soeurs. Même si cette pratique est citée comme datant de la fin du siècle, on peut supposer qu'elle a pu subsister pendant quelques décennies.

Il est sous-entendu que les enfants de 10 à 13 ans avaient l'habitude de prendre soin des enfants qui étaient trop jeunes pour fréquenter les salles d'asile [sorte de garderie, T.H.]. Les registres de l'école paroissiale St-Joseph que dirigeaient les Soeurs de la Congrégation de Notre-Dame indiquent que la majorité des enfants quittaient

\footnotetext{
14 Les manufactures de coton touchent 1123 enfants au-dessous de seize ans en 1901 et 979 en 1916. - Gouvernement du Canada, Recensement du Canada (Ottawa, 1901), III: 33-37 (pour les manufactures employant plus de 100 personnes) et idem, Recensement postal des manufactures (Ottawa, 1916): 115-119.

15 Gouvernement du Canada, Recensement du Canada (Ottawa, 1921), IV: ci et cii.
} 
l'école après la troisième année mais qu'à cet âge, vraisemblablement 10 ou 11 ans, très peu allaient travailler. Il y avait certainement un nombre considérable d'enfants disponibles pouvant garder les enfants plus jeunes dans leur propre famille ou possiblement chez les voisins, libérant la mère qui pouvait alors travailler à l'extérieur de la maison. ${ }^{16}$

Mais plus important encore, l'exclusion dans les recensements du travail à la ferme (sauf lorsqu'il est considéré comme occupant la majeure partie du temps) ${ }^{17}$ est d'une extrême importance au Québec où le type d'agriculture est caractérisé par la prépondérance de la ferme familiale d'auto-subsistance. Dans ce type de ferme où il n'y a aucun travailleur salarié, toute la famille participe à la production, ce qui rend le travail des enfants particulièrement nécessaire. Or ce type d'agriculture est resté dominant au Québec jusqu'à la deuxième guerre mondiale. Il s'ensuit qu'une plus grande proportion d'enfants de quatorze ans et moins était susceptible de travailler à la ferme au Québec, durant une partie suffisante de l'année scolaire pour que la fréquentation en soit fortement compromise.

De plus, il n'était pas rare l'automne, que le père agriculteur parte dans les camps de búcherons avec ses garçons de douze, treize et quatorze ans, pour constituer une équipe de chantier. À cet égard, l'Union catholique des cultivateurs (UCC) exigeait dans les années trente la réglementation du travail des enfants dans les chantiers.

De plus, les recensements ne tiennent pas compte de tout le travail effectué en dehors des heures de classe. Que l'on pense à la vente des journaux, au travail dans les commerces, aux jeunes livreurs(euses), au travail des messagers, etc., bref à toutes ces activités qui alourdissaient la journée des enfants et qui risquaient de les rendre peu disposés(es) à affronter une autre journée de classe.

Le travail des enfants occupait donc une plus grande partie de la population de dix à quatorze ans que ne le laissent supposer les recensements fédéraux et ceci sans compter les infractions et la non-application des lois.

En plus de tous les échappatoires contenus dans la loi (dont nous avons parlé plus haut), celle-ci était très difficile à appliquer. Le nombre d'inspecteurs était insuffisant, trois en 1888 pour couvrir l'ensemble du territoire. En 1918, Louis Guyon, inspecteur en chef des fabriques disait:

L'énorme augmentation de la fabrication dans la ville de Montréal, depuis le commencement de la guerre, ne nous permet de faire que

16 Suzanne D. Cross, «La majorité oubliée: le rôle des femmes à Montréal au $19^{\mathrm{e}}$ siècle», dans M. Lavigne, Y. Pinard, Les femmes dans la société québécoise (Montréal, éd. du Boréal Express, 1977), 50.

Ce que les parents ne déclaraient pas toujours. 
quelques courtes visites dans les grands centres du district, exception faite cependant pour la ville de Hull et les centres importants sur les bords de la rivière Ottawa, entre Montréal et Aylmer. ${ }^{18}$

De plus, les inspecteurs avaient très peu de pouvoirs. En 1921, Guyon écrivait:

A good number of children 14 and under will escape all control [...] because our inspectors have not always jurisdiction in the places where they are employed. ${ }^{19}$

Ainsi, Alfred Robert, inspecteur des fonderies de Montréal explique, en 1918:

Dans notre province, la loi fixe l'âge d'admission des jeunes filles et des garçons dans la fabrique à 14 ans. Il est pénible de constater que des enfants au-dessous de l'âge réglementaire sont employés dans les fabriques; souvent l'on constate, après enquête, que le certificat d'âge présenté à 1 'industriel a été rempli et signé par l'enfant, et le patron se croit justifiable, sur la présentation d'un tel certificat d'âge, de donner du travail à l'enfant nonobstant sa taille souvent petite et son état physique bien peu rassurant. ${ }^{20}$

De nombreux autres témoignages vont dans le même sens que celui-ci. Madame Émilia Cartier, qui était âgée de soixante-seize ans en 1972, montre les dures conditions d'emploi et les ruses des employeurs pour contourner la loi.

J'ai commencé à travailler à 13 ans à la Dominion Textile (en 1909). Dans le temps on appelait ça la facterie de coton. J'ai commencé à travailler à $\$ 3.50$ par semaine. Je commençais à 6 heures du matin et je finissais à 6 h15 le soir (i.e. une journée de plus de 10 heures). Le samedi après-midi, je travaillais jusqu'à 3 heures. Il fallait avoir 14 ans pour commencer à travailler là mais moi, ils me cachaient et j'ai toujours travaillé là. J'ai jamais lâché. ${ }^{21}$

En 1916, Guyon disait: «Child labour remains the same unsolvable problem we have encountered... since $1888 .{ }^{22}$

Sans loi de fréquentation scolaire et avec une législation sur le travail des enfants beaucoup plus permissive que dans les autres provinces, le Québec s'est caractérisé par une moins longue fréquentation scolaire que dans les autres provinces et par une concentration du travail

\footnotetext{
18 Gouvernement de la province de Québec, Rapport annuel du ministère des Travaux publics et du Travail de la province de Québec (1918), 69.

19 «Un bon nombre d'enfants de 14 ans et moins échappera à tout contrôle [...] car les endroits où ils travaillent ne tombent pas toujours sous la juridiction de nos inspecteurs (traduction de l'auteur)». T. Copp, op. cit., 54.

${ }^{20}$ Rapport annuel du Ministère des Travaux publics et du Travail... idem, 85-96.

21 M. Pelletier, Y. Vaillancourt, Les politiques sociales et les travailleurs (Montréal, Éd. de l'auteur, 1974), cahier I: 37.

22 «Le travail des enfants demeure le même problème insoluble que nous avons rencontré... depuis 1888 (traduction de l'auteur)». T. Copp, op. cit., 54.
} 
des enfants canadiens atteignant presque $50 \%$ certaines années. De plus, malgré l'importance du phénomène tel que recensé, on constate qu'il ne donne qu'un léger aperçu de l'utilisation de la jeune force de travail au Québec.

Ainsi, au Québec, l'utilisation du travail des enfants, tant dans les fabriques et usines, à la ferme, à la maison, dans les commerces, s'est donc poursuivie jusqu'en 1941 et même au-delà.

\subsection{Type d'industrialisation au Québec}

\subsection{Structure industrielle du Québec}

Le développement industriel du Québec se caractérise par la place privilégiée qu'occupe l'industrie légère et par l'importance des secteurs industriels à forte intensité de main-d'oeuvre. Par rapport à l'Ontario, la province de Québec se spécialise dans la production de biens de consommation alors que l'Ontario, elle, l'est dans l'industrie des biens de production. Le Québec dominait dans les industries légères (cigares et cigarettes, fil et tissu de coton, etc...) pendant que l'Ontario se spécialisait dans l'industrie lourde et les secteurs hautement mécanisés (automobiles, pneus, appareils mécaniques etc...). En 1952, le Québec concentre encore des secteurs à forte utilisation de main-d'oeuvre non qualifiée: tabac et produits du tabac $(93,5 \%$ de la valeur de la production canadienne); confection pour femmes $(68,5 \%)$; textiles synthétiques $(62,9 \%)$; filés et tissus de coton $(62,4 \%)$; chaussures en cuir $(60,0 \%)$; confection pour hommes $(56,5 \%)^{23}$.

Plus significative encore est la distribution de la population par secteur économique. En 1933, 1934 et 1939 l'industrie du textile concentre $30,0 \%$ de la main-d'oeuvre industrielle. On connaît les caractéristiques de ce type d'industrie: forte consommatrice de jeune force de travail et secteur industriel à faible besoin de force de travail scolarisée. En 1950, le textile, les chaussures en cuir et le tabac, autres secteurs réputés pour l'utilisation des enfants dans la production et pour le peu de développement technique, concentraient $35,4 \%$ du personnel des quarante principales industries au Québec et $25 \%$ de la main-d'oeuvre de toutes les industries.

La structure industrielle de Québec explique donc une partie du travail des enfants au Québec, mais aussi la faible incitation pour les capitalistes de ces secteurs, à privilégier une formation scolaire de la force de travail.

\footnotetext{
${ }^{23}$ Patrick Allen, Tableau de l'activité économique de la province de Québec (Commission royale d'enquête sur les problèmes constitutionnels, 1955), 210.
} 


\subsection{Type d'agriculture au Québec}

L'agriculture au Québec se caractérise par la ferme familiale d'autosubsistance. En effet, jusqu'à la seconde guerre mondiale, les revenus nets en nature sont plus élevés que les revenus nets monétaires (Tabieau 4).

TABLEAU 4

REVENU NET MONÉTAIRE ET EN NATURE EN POURCENTAGE DU REVENU NET TOTAL QUÉBEC, 1926-1944

\begin{tabular}{lcc}
\hline Année & $\begin{array}{c}\text { Revenu net en nature } \\
\%\end{array}$ & $\begin{array}{c}\text { Revenu net monétaire } \\
\%\end{array}$ \\
\cline { 2 - 2 } & 64 & 36 \\
1926 & 66 & 34 \\
1927 & 61 & 39 \\
1928 & 65 & 35 \\
1929 & 76 & 24 \\
1930 & 83 & 17 \\
1931 & 96 & 4 \\
1932 & 98 & 2 \\
1933 & 77 & 23 \\
1934 & 76 & 24 \\
1935 & 64 & 36 \\
1936 & 61 & 39 \\
1937 & 59 & 41 \\
1938 & 52 & 48 \\
1939 & 48 & 52 \\
1940 & 48 & 52 \\
1941 & 46 & 54 \\
1942 & 44 & 56 \\
1943 & 39 & 61 \\
1944 & 53 & \\
\hline
\end{tabular}

SOURCE: Gouvernement du Canada, Statistique Canada, Cansim 200134 à 41 (mat. 267).

En 1931, encore $30 \%$ de la main-d'oeuvre totale travaillent en agriculture. Cette main-d'oeuvre est composée à $50 \%$ par les cultivateurs exploitants, à $40 \%$ par les membres de la famille; il ne reste que $10 \%$ pour la main-d'oeuvre engagée. À cet égard, George V Haythorne $(1960)^{24}$ nous montre que c'est au Québec qu'il y a le plus de maind'oeuvre familiale non-payée. Même si l'auteur ne tient compte que de la population de quatorze ans et plus à partir de 1921, il nous permet de faire l'hypothèse qu'une partie des enfants de 12,13 et 14 ans étaient employés aux travaux de la ferme et pour une bonne partie de l'année.

\footnotetext{
24 George V Haythorne, Labour in Canadian Agriculture, (Cambridge, Harvard University Press, 1960), 27.
} 
L'agriculture au Québec dépend donc de la main-d'oeuvre familiale absolument indispensable à sa survie.

\section{Conclusion}

Compte tenu des faits que nous venons de montrer, nous croyons que le travail des enfants au Québec a été un des facteurs expliquant le retard de l'obligation scolaire. Patrice Grevet (1976) ${ }^{25}$ démontre d'ailleurs que l'avènement de l'obligation scolaire en France est lié à la résolution de deux contradictions pour la classe capitaliste: la contradiction entre le besoin de force de travail scolarisée et les frais d'entretien de cette force de travail; celle entre le besoin de force de travail scolarisée et les avantages de l'exploitation des enfants. Contrairement à ce que l'on aurait pu croire, ces facteurs ont aussi joué un rôle au Québec, quelque soixante ans plus tard, dans un contexte historique totalement différent. Il va sans dire que ce facteur n'intervient pas avec la même ampleur dans ces deux formations sociales.

Au Québec, en plus d'être l'enjeu d'une lutte entre fractions de la bourgeoisie, le travail des enfants consistait surtout en un facteur essentiel de la survie de la ferme familiale d'auto-subsistance, principal type d'agriculture de cette province. Des nécessités économiques ont donc retenu les enfants soit sur la ferme, soit à la maison, soit dans les manufactures, les commerces, la rue, etc. Pour l'agriculteur, le travail de sa femme et de ses enfants est indispensable à sa survie, au moins pour une partie de l'année. Pour l'ouvrier, les salaires de famine obligent les enfants à subvenir aux besoins de la famille. Mais, inversement, la présence des enfants sur le marché du travail fait concurrence à la maind'oeuvre adulte et maintient le salaire du père très bas, celui des enfants étant considéré (tout comme celui de la femme d'ailleurs!) comme un salaire d'appoint.

Ainsi, quoique le mouvement ouvrier ait lutté depuis fort longtemps contre l'exploitation du travail des enfants ${ }^{26}$ (et à cet égard, cette lutte à suscité une beaucoup plus grande unanimité que celle de l'obligation scolaire), il existait une contradiction entre la position du mouvement et les dures nécessités économiques de chaque famille.

La structure industrielle du Québec et le type d'agriculture qui y prédomine nous font comprendre pourquoi le Québec concentre le travail des enfants canadiens. De plus, la très grande importance de l'industrie légère et plus particulièrement la prédominance du textile, de même que certains secteurs comme le tabac, la chaussure, laissent présager qu'une fraction de la bourgeoisie n'avait pas du tout intérêt à ce qu'une loi de fréquentation scolaire obligatoire soit votée, l'instruction minimum généralisée étant pour elle à toute fin pratique inutile.

\footnotetext{
25 Patrice Grevet, Besoins populaires et financement public (Paris, Éditions sociales, 1976), 543 pages.

Voir à ce sujet les chapitres $6,7,8,9$ et 10 de notre thèse qui portent spécifiquement sur les positions du CMTC, de la CTCC et de l'UCC sur le travail des enfants et l'instruction obligatoire.
} 